\title{
A utilização da atenção primária à saúde por imigrantes em Florianópolis
}

\author{
The use of primary health care by immigrants in Florianopolis

\section{La utilización de la atención primaria a la salud por inmigrantes en Florianópolis}

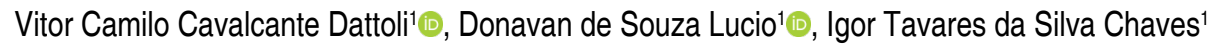 \\ ${ }^{1}$ Prefeitura Municipal de Florianópolis. Florianópolis, SC, Brasil.
}

\section{Resumo}

Objetivo: Conhecer o perfil do imigrante que utiliza a atenção primária de Florianópolis. Métodos: Estudo transversal de base populacional com dados cadastrais de estrangeiros moradores de Florianópolis, que utilizaram a Atenção Primária à Saúde entre os anos de 2014 e 2016. As variáveis utilizadas foram: nacionalidade, sexo, idade, nível de escolaridade, raça, endereço de moradia e tipos de serviços utilizados. Resultados: Um total de 10.108 estrangeiros utilizaram a atenção básica em Florianópolis no período do estudo, com predomínio de adultos, brancos, com ensino médio ou superior, predominância de argentinos, uruguaios e haitianos, utilizando predominantemente serviços da farmácia e consultas médicas, distribuídos em todas unidades de saúde da capital. Houve maior nível de escolaridade e maior proporção de crianças e idosos na população provinda de países desenvolvidos, além de uma menor utilização dos serviços. Discussão: Estudo pioneiro no Brasil. O perfil dos imigrantes estudados é comparável à população de estudos europeus, com predomínio de latino-americanos e caribenhos, com faixa etária prevalecendo população economicamente ativa, utilizando consultas médicas e com alta escolaridade entre imigrantes de países desenvolvidos.

Palavras-chave: Emigrantes e Imigrantes; Atenção Primária à Saúde; Perfil de Saúde

\begin{abstract}
Objective: To know the immigrant's profile who use primary care in Florianopolis. Methods: Cross-sectional populational-based study with register data from foreigners who used Primary Health Care in Florianopolis between 2014 and 2016. The variables were nationality, sex, age, level of education, race and service used. Results: A total of 10,108 immigrants accessed primary care in Florianopolis during the study period, with a predominance profile of adults, Caucasian, with medium/high level of education, Latin Americans, mainly using pharmacy and medical consultations, distributed in all primary care units at Florianopolis. We observed a higher level of schooling and a greater proportion of children and elderly people in the population coming from developed countries, in addition to a lower use of services. Discussion: The profile of immigrants in the study population is comparable to the immigrant population in some European studies, with a predominance of Latin Americans and Caribbean, with a high frequency of economically active age group, using medical consultations and high education among immigrants from developed countries.
\end{abstract}

Keywords: Emigrants and Immigrants; Primary Health Care; Health Profile

Como citar: Dattoli VCC, Lucio DS, Chaves ITS. A utilização da atenção primária à saúde por imigrantes em Florianópolis. Rev Bras Med Fam Comunidade. 2019;14(41):1786. http://dx.doi.org/10.5712/rbmfc14(41)1786
Autor correspondente: Vitor Camilo Cavalcante Dattoli. E-mail: vdattoli@hotmail.com Fonte de financiamento: declaram não haver.

Parecer CEP:

2.453.492 (SES/SC), aprovado em 21/12/2017.

Procedência e revisão por pares: revisado por pares. Recebido em: 29/06/2018. Aprovado em: 06/11/2018. 


\section{Resumen}

Objetivo: Conocer el perfil del inmigrante que utiliza la atención primaria a la salud de Florianópolis. Métodos: Estudio transversal de base poblacional con datos de registro de extranjeros que utilizaron la Atención Primaria a la Salud de Florianópolis entre los años 2014 y 2016 . Las variables utilizadas fueron: nacionalidad, sexo, edad, nivel de escolaridad, raza, dirección de vivienda y residencia tipos de servicios utilizados. Resultados: Total de 10.108 inmigrantes utilizaron la atención básica en Florianópolis en el período del estudio, con un perfil de predominio de adultos, blancos, con enseñanza media o superior de escolaridad, predominio de argentinos, uruguayos y haitianos, utilizando predominantemente servicios de la farmacia y consultas médicos, distribuidos en todas las unidades de salud de la capital. Se observó un mayor nivel de escolaridad y mayor proporción de niños y ancianos en la población proveniente de países desarrollados, además de una menor utilización de los servicios. Discusión: Estudio pionero en Brasil, el perfil de los inmigrantes en la población del estudio es comparable a la población de estudios europeos, con predominio de latinoamericanos y caribeños, con rango de edad prevaleciendo población económicamente activa, utilizando consultas médicas y con alta escolaridad entre inmigrantes de países desarrollados.

Palabras clave: Emigrantes e Inmigrantes; Atención Primaria de Salud; Perfil de Salud

\section{Introdução}

A migração internacional é atualmente considerada um grande desafio em nível global. Ao longo da nossa história, vem se observando o impacto da imigração ao redor do mundo, levando a mudanças irreversíveis. ${ }^{1}$ Em 2017, a Organização das Nações Unidas (ONU) estimou que 1 em cada 29 pessoas vivia como estrangeiros, correspondendo a cerca de $3,4 \%$ da população mundial. ${ }^{2}$ Neste cenário, o Brasil representa um importante destino migratório na região latino-americana.

Dados do Instituto Brasileiro de Geografia e Estatística (IBGE) de 2010 mostram que o número de imigrantes aumentou 86,7\% em dez anos. Segundo o IBGE, em 2010, 286.468 imigrantes viviam há pelo menos 5 anos no Brasil - no ano de 2000 eram 143.644. ${ }^{3} \mathrm{O}$ Brasil abriga 1.847.274 imigrantes regulares, segundo estatísticas da Polícia Federal atualizadas em março de 2015. Conforme a classificação adotada pela instituição, esse total engloba 1.189 .947 permanentes; 595.800 temporários; 45.404 provisórios; 11.230 fronteiriços; 4.842 refugiados; e 51 asilados, representando 0,9\% da população brasileira. ${ }^{4}$

Dessa forma, a atual ampliação dos fluxos migratórios internacionais coloca em debate a responsabilização dos estados pela garantia de direitos sociais básicos, dentre eles, o acesso à saúde. $O$ acesso e o status de saúde desta população são marcadores fundamentais da integração dos imigrantes. $O$ grande exemplo deste desafio é "efeito do imigrante saudável". Diversos estudos mostram que imigrantes, nos primeiros anos de mudança, são mais saudáveis do que a população nativa, e que ao longo do tempo, sua saúde tende a deteriorar. ${ }^{5-7}$ Tal fenômeno é explicado pelo perfil de imigrantes jovens e saudáveis, que tendem a adoecer ao longo da sua estadia no exterior, devido a situações socioeconômicas e culturais adversas por ser um imigrante. ${ }^{8}$

Em Florianópolis, SC, a atenção primária tem passado por desafios no atendimento da população estrangeira. A falta de conhecimento da demanda de pacientes imigrantes, a chegada constante de novas pessoas para o território, dificuldades com idiomas e divergências culturais costumam exigir consultas com maior tempo e atenção, tendendo a sobrecarregar as equipes de saúde da família (dados não publicados).

Desta forma, conhecer a população imigrante pode permitir uma melhor organização do sistema de saúde e das equipes de saúde da família, proporcionando a oferta de serviços de saúde mais humanos, integrais e com maior equidade. 


\section{Métodos}

\section{Desenho e população do estudo}

Trata-se de estudo transversal de base populacional, com utilização de dados secundários provenientes de fichas de cadastro no INFOSAÚDE, software utilizado como prontuário eletrônico no município de Florianópolis.

A população do presente estudo foi composta por pessoas não brasileiras (nacionalidade não brasileira no cadastro), que possuíam cadastros ativos no Florianópolis e utilizaram a atenção primária à saúde (APS) de Florianópolis no período de janeiro de 2014 a dezembro de 2016.

Foram excluídos os estrangeiros que, apesar de utilizarem a APS no período do estudo, não possuíam endereço de moradia no seu cadastro (total de 11.173), os quais possivelmente utilizaram o serviço como não residentes de Florianópolis, por exemplo, turistas e/ou atendimento nas UPAs (unidades de pronto-atendimento). Vale ressaltar que a utilização do termo imigrante no trabalho é justificada pela ideia de que apenas aqueles que se declararam moradores de Florianópolis foram incluídos no estudo, apesar da limitação de que moradores temporários e pessoas com endereços de amigos e familiares possam ter entrado no estudo.

\section{Análise de dados}

As variáveis utilizadas foram idade, sexo, escolaridade, nacionalidade, raça, tipo de serviço utilizado, endereço de moradia. A determinação do perfil socioeconômico foi classificada conforme a ONU, em 2016, nomeadas no presente trabalho como países desenvolvidos e em desenvolvimento. ${ }^{9} \mathrm{Em}$ relação à variável raça, é uma informação de autodeclaração do usuário do sistema no momento do cadastro.

Os dados epidemiológicos foram codificados, digitados em banco de dados e analisados utilizando-se o software SPSS versão 20.0. Uma estatística descritiva foi feita das variáveis primárias incluídas no estudo. Para análise de estratificação, de acordo com status socioeconômico dos países, a associação entre escolaridade, idade e serviços utilizados foi estimada por meiode Odds Ratio e Intervalo de Confiança com 95\% de significância. O modelo multivariável foi ajustado por sexo, idade, raça e centro de sáude. Estas variáveis foram utilizadas para ajuste por serem consideradas pontenciais confundidoras.

\section{Considerações Éticas}

O presente estudo obteve aprovação ética pelo Comitê de Ética em Pesquisa da Secretaria Estadual de Saúde de Santa Catarina (parecer número: 2.453.492).

\section{Resultados}

Entre janeiro de 2014 a dezembro de 2016, 10.108 imigrantes foram atendidos na atenção primária em Florianópolis, representando $2,20 \%$ do total da população (463.470 pessoas) atendida na APS no município neste período. Destes, 5.500 usuários (54,4\%) eram do sexo masculino e 4.608 (45,6\%) feminino, predominantemente brancos $7.994(79,1 \%)$, seguidos de pessoas da cor preta $1.455(14,4 \%)$ e parda 
$521(5,1 \%)$, respectivamente. Em relação à faixa etária, 1.254 (12,4\%) tinham até 17 anos, 7.764 (76,8\%) entre 18 e 59 anos e 1.090 (10,8\%) tinham 60 anos ou mais de idade. Avaliando o nível de escolaridade da população, $2.097(20,7 \%)$ eram analfabetos ou analfabetos funcionais, $1.338(13,2 \%)$ estudaram até ensino fundamental, $3.388(33,7 \%)$ até ensino médio e $3.137(31,0 \%)$ tinham nível superior ou alguma pós-graduação (Tabela 1).

Tabela 1. Perfil dos imigrantes que utilizaram a Atenção Primária em Florianópolis, entre 2014-2016.

\begin{tabular}{|c|c|c|}
\hline Variável ( $\mathrm{N}=10.108)$ & $\mathbf{n}$ & $(\%)$ \\
\hline \multicolumn{3}{|l|}{ Sexo } \\
\hline Feminino & 4.608 & 45,6 \\
\hline Masculino & 5.500 & 54,4 \\
\hline \multicolumn{3}{|l|}{ Cor } \\
\hline Preta & 1.455 & 14,4 \\
\hline Parda & 521 & 5,1 \\
\hline Branca & 7.994 & 79,1 \\
\hline Indígena & 36 & 0,4 \\
\hline Amarela & 102 & 1,0 \\
\hline \multicolumn{3}{|l|}{ Faixa etária } \\
\hline $0-17$ anos & 1.254 & 12,4 \\
\hline $18-59$ anos & 7.764 & 76,8 \\
\hline$\geq 60$ anos & 1.090 & 10,8 \\
\hline \multicolumn{3}{|l|}{ Escolaridade } \\
\hline Analfabeto/Alfabetizado & 2.097 & 20,7 \\
\hline Ensino Fundamental & 1.338 & 13,2 \\
\hline Ensino Médio & 3.388 & 33,7 \\
\hline Superior/Especialização & 3.137 & 31,0 \\
\hline \multicolumn{3}{|l|}{ Países (170) } \\
\hline Em desenvolvimento & 7.566 & 74,9 \\
\hline Desenvolvidos & 2.542 & 25,1 \\
\hline \multicolumn{3}{|l|}{ Continentes } \\
\hline América do Sul & 5.363 & 53,1 \\
\hline América Central & 1.392 & 13,8 \\
\hline América do Norte & 463 & 4,6 \\
\hline Europa & 1.978 & 19,6 \\
\hline África & 337 & 3,3 \\
\hline Ásia & 498 & 4,9 \\
\hline Oceania & 77 & 0,8 \\
\hline
\end{tabular}

Em relação à nacionalidade, a população imigrante, que acessou a APS na capital entre 2014 e 2016, foi proveniente de 170 diferentes nacionalidades, provindas dos seis continentes, sendo destes $7.566(74,9 \%)$ de países em desenvolvimento e $2.542(25,1 \%)$ de países desenvolvidos. Como mostrado no Gráfico 1, a nacionalidade predominante foi a argentina, com 2.618 (25,9\%) pessoas acessando a APS no período do estudo, seguida dos uruguaios, com 1.264 (12,5\%), e haitianos, com $1.223(12,1 \%)$ pessoas, respectivamente. 


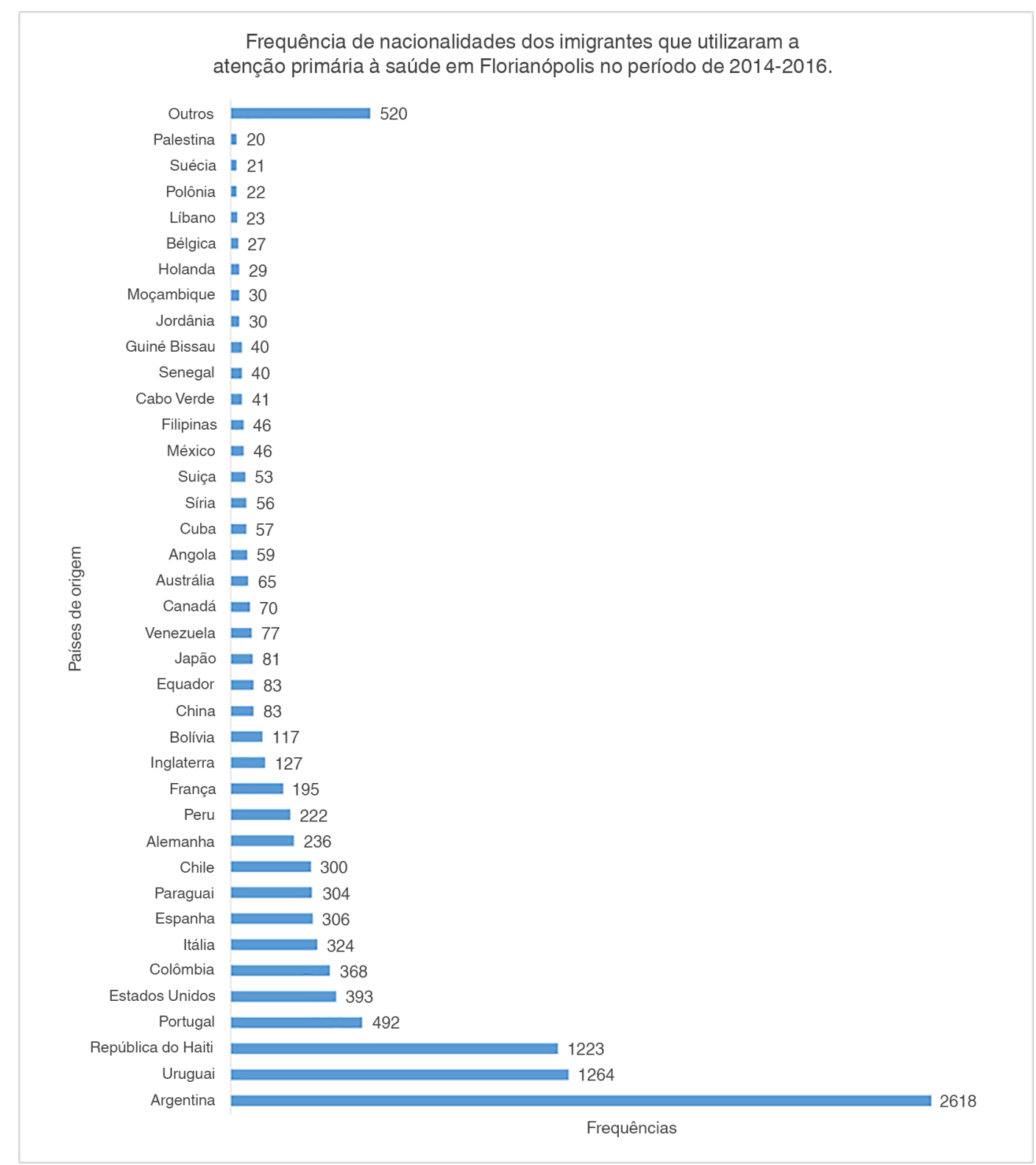

Gráfico 1. Frequência de nacionalidades dos imigrantes que utilizaram a atenção primária à saúde em Florianópolis no período de 2014-2016.

Avaliando a distribuição dos estrangeiros residentes em Florianópolis, a Figura 1 evidencia que todos os 49 centros de saúde possuem imigrantes como pacientes.

Dentre todos os CSs (Centros de Saúde) da capital, o CS Lagoa da Conceição foi a unidade mais utilizada por estrangeiros, com $863(8,5 \%)$ usuários estrangeiros, seguido do CS Canasvieiras $844(8,3 \%)$ e CS Ingleses 641 (6,3\%), respectivamente (Gráfico 2).

Em relação ao número de serviços ofertados na APS de Florianópolis, a dispensação de medicamentos pela farmácia foi o serviço mais procurado pela população do estudo, sendo utilizada por 6.301 (62,3\%) pessoas, seguido por atendimento médico, utilizado por $6.005(59,4 \%)$ das pessoas; ainda, os atendimentos 


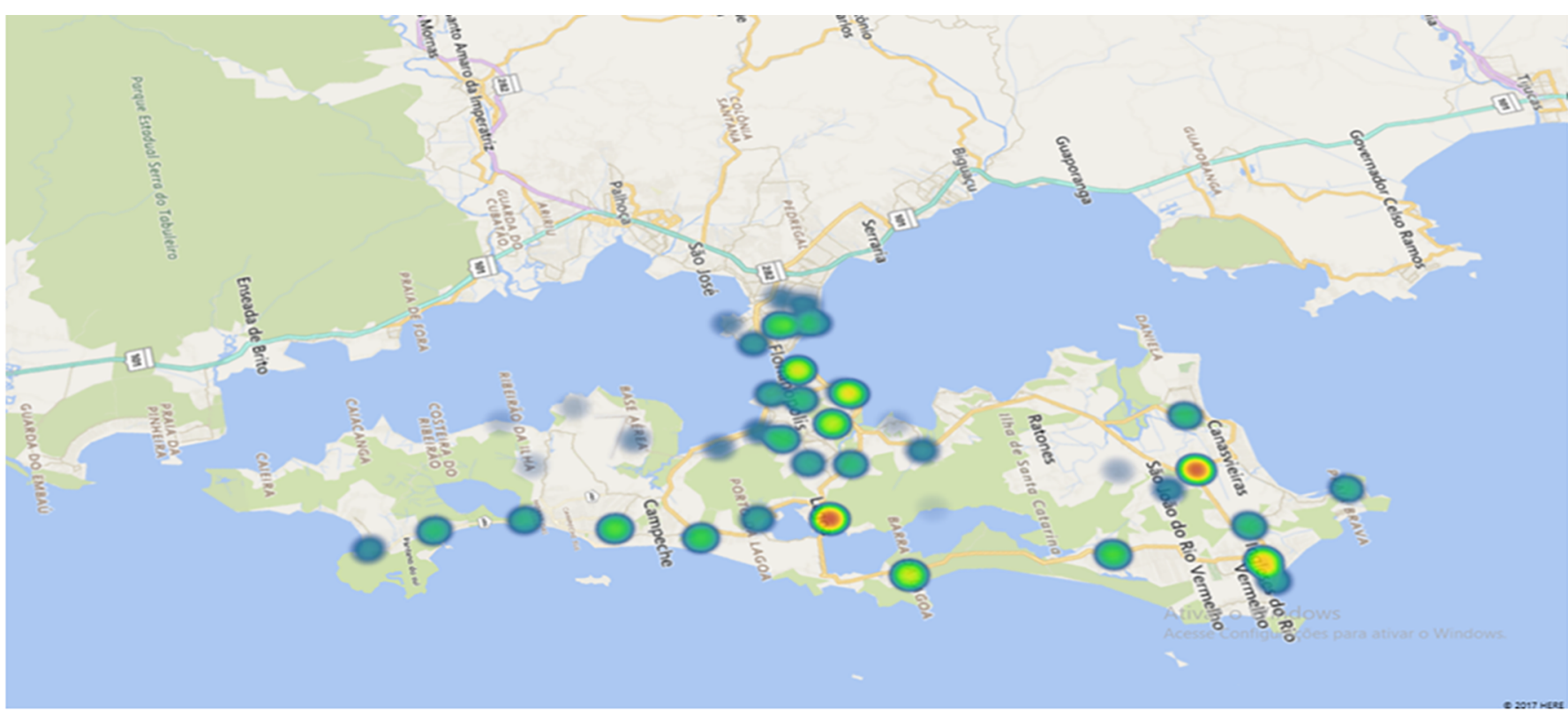

Figura 1. Distribuição dos imigrantes que utilizaram a Atenção Primária à Saúde em Florianópolis, de acordo com centro de saúde adscrito.

por técnicos de enfermagem, consulta de enfermagem e consulta odontológica foram contemplados por, respectivamente, $4.936(48,8), 5.588(55,3 \%)$ e 2.370 (23,4\%) usuários, com média de 2,39 serviços utilizados por pessoa.

Quando avaliamos comparativamente as populações provindas de países desenvolvidos e em desenvolvimento, observa-se que existe maior proporção de crianças/adolescentes ( $<18$ anos) - 20,9\% e idosos (>60 anos) - 18,1\% - na população de países desenvolvidos, em comparação com países em desenvolvimento, os quais possuem apenas $9,5 \%$ e $8,3 \%$, resultado estatisticamente significante (Tabela 2 ).

Ainda, como observado na Tabela 3, em relação à escolaridade, pessoas com nivel superior ou pós-graduação tiveram maior chance de serem provenientes de países desenvolvidos, enquanto estrangeiros com nível de escolaridade mais baixo, como ensino fundamental e médio, tiveram maior chance de serem provenientes de países em desenvolvimento.

Por fim, como visto na Tabela 4, praticamente todos os serviços ofertados na APS (dispensação de medicamentos, consultas médicas, de enfermagem e odontológica) foram proporcionalmente mais utilizados pela população provinda de países em desenvolvimento, sendo inversamente associado com a população proveniente de países desenvolvidos, com exceção de atendimento por técnicos de enfermagem, que não apresentou resultados com diferença estatística. 


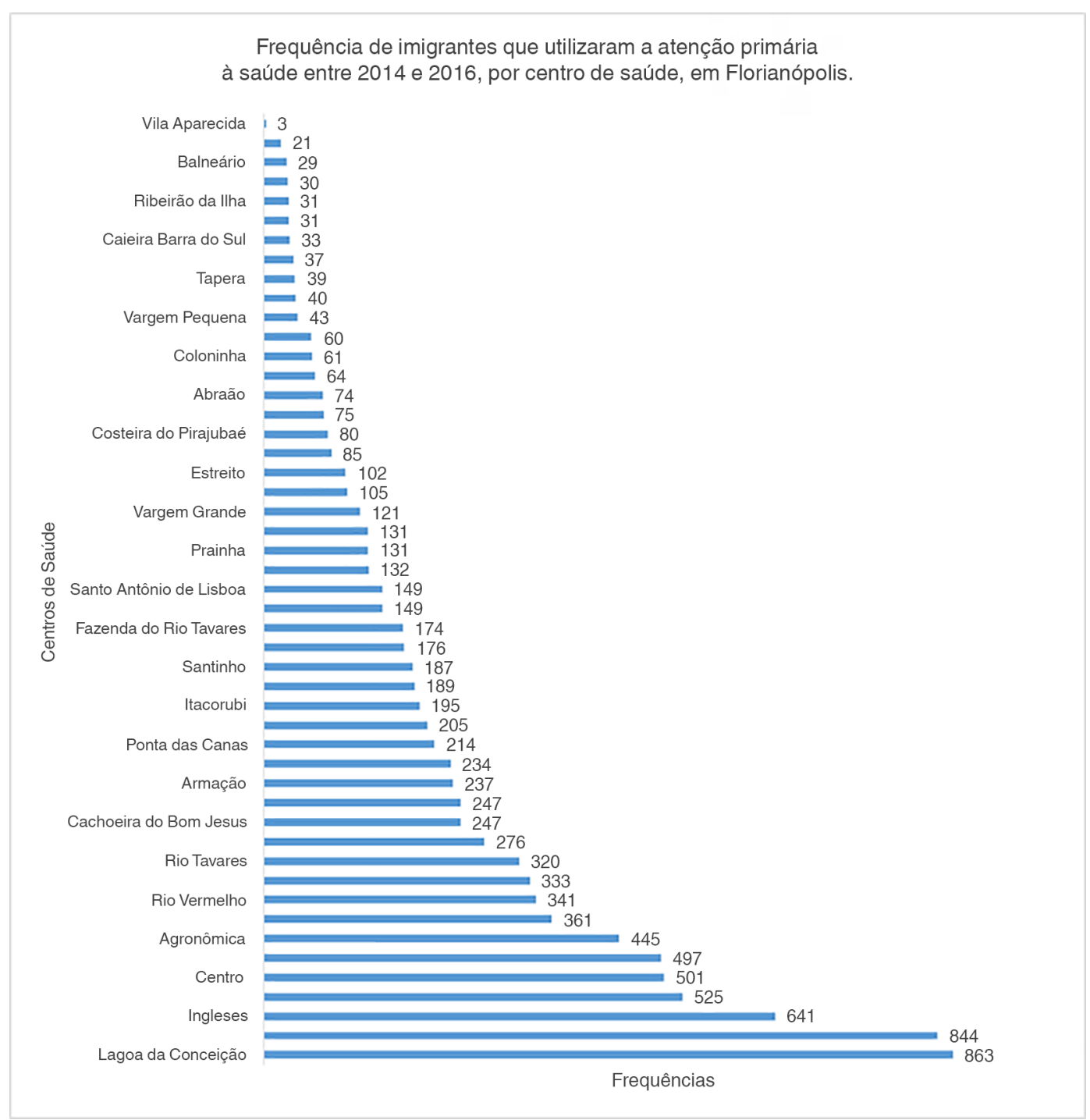

Gráfico 2. Frequência de imigrantes que utilizaram a atenção primária à saúde entre 2014 e 2016, por centro de saúde, em Florianópolis.

Tabela 2. Nível econômico do país de origem conforme a idade dos imigrantes que utilizaram a atenção primária à saúde de Florianópolis, 2014-2016.

\begin{tabular}{lcccc}
\hline $\mathbf{n}=\mathbf{1 0 . 1 0 8}$ & Em desenvol. $(\mathbf{n = 7 . 5 6 6 )}$ & Desenvolvidos $(\mathbf{n = 2 . 5 4 2 )}$ & & \\
\hline Faixa etária & $\mathbf{n}(\%)$ & $\mathbf{n}(\%)$ & OR (IC 95\%) & OR (IC 95\%) \\
\hline$<18$ anos & $723(9,6)$ & $531(20,9)$ & $2,94(2,60-3,34)$ & $4,05(3,39-4,84)$ \\
$18-59$ anos & $6214(82,1)$ & $1550(61,0)$ & 1 & 1 \\
$>60$ anos & $629(8,3)$ & $461(18,1)$ & $2,94(2,57-3,35)$ & $3,04(2,62-3,54)$ \\
\hline
\end{tabular}

\# Regressão logistica bruta; ${ }^{\circledR}$ Regressão logistíca multivariável ajustada por sexo, escolaridade, raça e unidade de atendimento. 
Tabela 3. Nível econômico do país de origem conforme a escolaridade dos imigrantes que utilizaram a Atenção Primária à Saúde de Florianópolis, 2014-2016.

\begin{tabular}{lcccc}
\hline $\mathbf{n = 9 . 9 6 0}$ & Em desenvol. (n=7.461) & Desenvolvidos (n=2.499) & & \\
\hline Escolaridade & $\mathbf{n ~ ( \% )}$ & $\mathbf{n ~ ( \% )}$ & OR (IC 95\%) & OR (IC 95\%) \\
\hline Analfabetos/Alfabetizados & $1.524(20,4)$ & $573(22,9)$ & 1 & 1 \\
Ensino Fundamental & $1.093(14,6)$ & $245(9,8)$ & $0,60(0,50-0,71)$ & $0,67(0,55-0,82)$ \\
Ensino Médio & $2.808(37,6)$ & $580(23,2)$ & $0,55(0,48-0,63)$ & $0,75(0,63-0,89)$ \\
Superior/Especialização & $2.036(27,3)$ & $1.101(44,1)$ & $1,44(1,28-1,62)$ & $1,71(1,44-2,04)$ \\
\hline
\end{tabular}

\# Regressão logistica bruta; \& Regressão logistíca multivariável ajustada por sexo, idade, raça e unidade de atendimento.

Tabela 4. Padrão de utilização da atenção primária à saúde, por imigrantes, de acordo com status socioeconômico do país de origem, Florianópolis 2014-2016.

\begin{tabular}{|c|c|c|c|c|}
\hline$n=10.108$ & Em desenvol. $(n=7.566)$ & Desenvolvidos $(n=2.542)$ & & \\
\hline Serviço* & n (\%) & n (\%) & OR (IC 95\%) $)^{*}$ & OR (IC 95\%) $)^{2}$ \\
\hline Farmácia & $5.016(66,3)$ & $1.285(50,6)$ & $0,52(0,47-0,57)$ & $0,63(0,57-0,70)$ \\
\hline Técnico Enf. & $4.164(55,0)$ & $1.424(56,0)$ & $1,04(0,95-1,14)$ & $0,95(0,86-1,06)$ \\
\hline Enfermagem & $3.858(51,0)$ & $1.078(42,4)$ & $0,71(0,65-0,78)$ & $0,71(0,64-0,79)$ \\
\hline
\end{tabular}

\section{Discussão}

Em um consenso europeu, especialistas identificaram prioridades para um melhor sistema de saúde aos imigrantes entre elas: 1 - acesso fácil e equitativo aos cuidados de saúde, 2 - capacitação dos migrantes, 3 - serviços de cuidados de saúde culturalmente sensíveis, 4 - qualidade dos cuidados, 5 comunicação do paciente/prestador de cuidados de saúde, entre outros. Desta forma, conhecer melhor nossos imigrantes poderá favorecer tais prioridades. ${ }^{10} \mathrm{Em}$ uma realidade de APS forte e bem estruturada, como em Florianópolis, o atendimento à população imigrante/estrangeira representa um desafio para profissionais e sistema de saúde.

As análises de frequência mostraram uma população equilibrada quanto ao sexo biológico, uma predominância da faixa etária de adultos economicamente ativos, com nível de escolaridade mais alto que a média da população brasileira, provenientes principalmente da América Latina, distribuída por toda Florianópolis. Ainda, avaliando a escolaridade, um terço das pessoas do estudo cursavam ou tinham completado o nível superior, evidenciando um perfil de alta escolaridade dos estrageiros que acessam a atenção primária em Florianópolis. Segundo dados do IBGE, ${ }^{11}$ em 2015, 46,4\% dos brasileiros tinham, pelo menos, terminado o ensino médio, enquanto na população do estudo $60,8 \%$ haviam completado.

Em relação à nacionalidade, existiu o predomínio da população latino-americana/caribenha, com cerca de $65 \%$ do total da população do estudo. Mesmo perfil do estudo de Gimeno-Feliu et al., ${ }^{12}$ na Espanha, que observaram $56 \%$ de imigrantes latinos na atenção primária espanhola. Discriminando por países, verifica-se predomínio de argentinos e uruguaios; fato já esperado devido à proximidade geográfica de ambos países e de intenso fluxo turístico existente entre os dois países e a capital catarinense. 
Os haitianos representaram a $3^{a}$ nacionalidade predominante na atenção primária, fato este explicado pelo intenso fluxo migracional destes para diversos países latino-americanos, principalmente o Brasil, ${ }^{13}$ justificado, principalmente, pelo terremoto que devastou o país em 2010 e liderança brasileira nas missões de paz, apresentando o Brasil como um destino seguro e promissor para estas pessoas. ${ }^{14}$

Em relação à distribuição da população na capital catarinense, foi observado predomínio de pessoas vivendo na região ilhéu de Florianópolis, com apenas 10\% dos imigrantes vivendo na região continental. Os centros de saúde Lagoa da Conceição, Canasvieiras e Ingleses foram os centros de saúde com maior número de pessoas, resultados compatíveis com o observado no dia-a-dia, uma vez que são bairros preferidos por comunidade argentina (Canasvieiras e Ingleses) e por estrangeiros em geral (Lagoa da Conceição).

Em relação aos serviços utilizados, a dispensação de medicações foi o serviço mais utilizado, seguido de atendimento médico e por técnicos de enfermagem, respectivamente. Valendo ressaltar que em Florianópolis a dispensação de medicamentos pela APS é universal, qualquer morador, portando uma prescrição de medicamento, proveniente de serviços públicos ou privados, terá medicação ofertada. $\mathrm{Na}$ Espanha, na cidade de Zaragoza, Gimeno-Feliu et al. ${ }^{12}$ observaram que atendimentos médicos, de enfermagem e exames complementares foram os serviços mais utilizados na APS espanhola no ano de 2007. Apesar de não ter sido avaliado no presente estudo, diversas pesquisas mostram uma predileção dos imigrantes por serviços de emergência em relação à sua equipe de saúde da família. ${ }^{15,16}$ De Luca et al. ${ }^{17}$ cogitaram que a emergência é mais fácil de ser acessada pelos imigrantes, e fornecem uma solução imediata para suas necessidades de cuidados de saúde, com reduzida barreira linguística, cultural e burocrática, corriqueiras ao tentar acessar os serviços de saúde comuns.

Ao compararmos os perfis demográficos e de utilização da APS por imigrantes de acordo com o status socioeconômico de seus países, observamos predomínio de crianças/adolescentes e idosos na população provinda de países desenvolvidos, em comparação com países em desenvolvimento. Estes resultados sugerem um diferente padrão de imigração entre os dois grupos. Predomínio de pessoas economicamente ativas, provindas de países em desenvolvimento, ao passo que as pessoas oriundas de países desenvolvidos possuem maior estrutura migratória, com maiores garantias ao migrar, permitindo uma migração familiar.

Assim como em nosso estudo, Carrasco-Garrido et al. ${ }^{18}$ observaram, na Espanha, que "imigrantes econômicos", expressão utilizada pelos autores para imigrantes de países em desenvolvimento, que migram em busca de melhores condições socioeconômicas, tinham um predomínio de faixa etária de adultos economicamente ativos, ao passo que este predomínio se diluía para jovens e idosos quando os imigrantes proviam de outros países europeus, Canadá e Estados Unidos. No mesmo sentido, em estudo conduzido na Noruega, Diaz et al. ${ }^{19}$ viram que a faixa etária de imigrantes era diretamente proporcional ao nível socioeconômico do seu país de origem: quanto mais rico o país, maior a proporção de idosos na população imigrante, e divergindo, em partes, com os resultados do presente trabalho, que observou predomínio dos extremos de idade para países desenvolvidos.

Comparando a escolaridade de acordo com o nível socioeconômico dos países de origem da população imigrante, observamos maior proporção de pessoas com nível superior (completo ou incompleto) provindas de países desenvolvidos, evidenciando um maior nível de instrução dsta população. Em consonância com 
este achado, o estudo de Diaz et al. ${ }^{19}$ observou menor nível educacional em população provinda de países mais pobres em comparação com países com maior status socioeconômico. Entretanto, na Espanha, no estudo de Carrasco-Garrido et al., ${ }^{18}$ apesar de maior proporção de nível superior entre os imigrantes de países mais ricos, esta diferença não foi estatisticamente significante.

Avaliando o padrão de utilização dos serviços da APS, encontramos que pessoas provenientes de países em desenvolvimento tiveram uma maior utilização de quase todos os serviços analisados no presente estudo, com exceção dos serviços de técnicos de enfermagem (vacinas, curativos, entre outros). Atribuímos tal achado ao serviço de imunização realizado pelos profissionais técnicos de enfermagem, uma vez que as vacinas são universalmente utilizadas pela população brasileira no SUS e ofertada na APS.

Em relação ao número de consultas médicas, diferentemente do encontrado em nosso estudo, Diaz et al. ${ }^{19}$ não observaram diferenças significativas em relação à procura por consulta médica de acordo com o status econômico do país de origem. Já Gimeno-Feliu et al., ${ }^{12}$ observaram que populações provindas da América Latina e África utilizaram mais os serviços médicos (médico de família e pediatra), ao passo que europeus e norte-americanos (Canadá e Estados Unidos) fizeram mais uso de atendimento de enfermagem e exames complementares.

Ainda, em concordância com este achado, Soler-González et al., ${ }^{20}$ em uma cidade espanhola com 5 unidades de atenção primária, verificaram maior quantidade de visitas às unidades por parte da população africana, seguida do latino-americanos. Estratificando por serviço, observaram que os imigrantes utilizam mais serviços médicos, exames complementares e serviços odontológicos, ao passo que os espanhois utilizaram mais os atendimentos de enfermagem. Avaliando a utilização dos serviços da APS por crianças imigrantes na Noruega, a coorte de Fadnes et al. ${ }^{21}$ mostrou um maior uso por parte de populações provindas de países mais pobres em comparação com crianças não imigrantes ou imigrantes de países desenvolvidos.

Apesar do pioneirismo no cenário brasileiro e da escassa literatura mundial, é importante ressaltar que o estudo apresenta limitações metodológicas que dificultam maiores generalizações. Além de ser um estudo transversal, utilizou dados secundários, oriundos do prontuário eletrônico. A variável escolaridade é um dado coletado apenas no cadastro do usuário, e não é atualizada ao longo do tempo. Importante informar, também, que, nos cadastros, caso a cor da pessoa não seja referida ou perguntada na realização do seu cadastro, o sistema preenche automaticamente com a opção "branca". Assumimos a limitação de possível superestimação da população imigrante, uma vez que possíveis cadastros de pessoas não residentes ou residentes temporários possam ter sido criados, contabilizando-os como parte da população do presente estudo.

Além disso, a ausência de informações como tempo de estadia, fluência para o português e motivo de migração, informações não contempladas no presente estudo, dificultam maiores conclusões.

\section{Conclusões}

Apesar das limitações, este estudo é pioneiro na busca por entendimento da utilização de imigrantes e estrangeiros nos serviços públicos de saúde no Brasil. Pesquisar a respeito de imigração e saúde, entendendo melhor o uso dos serviços de saúde e suas relações com nível socioeconômico, carga de doença e tempo de moradia no país anfitrião, é uma condição essencial em prol de um serviço de saúde 
mais apropriado e acessível para a população imigrante. Logo, o melhor conhecimento da população estrangeira imigrante se faz necessário para a oferta de um serviço mais preparado e qualificado. Neste sentido, contribuímos para o entendimento de quem são os imigrantes que utilizam a APS, possibilitando, de alguma forma, uma melhoria na oferta dos serviços de saúde.

\title{
Contribuição dos autores
}

Concepção e/ou delineamento do estudo: VCCD, ITSC; Aquisição, análise ou interpretação dos dados: VCCD, DSL; Redação preliminar: VCCD; Revisão crítica da versão preliminar: VCCD, DSL.

Todos os autores aprovaram a versão final e concordaram com prestar contas sobre todos os aspectos do trabalho.

\section{Conflito de interesses}

\author{
Declaram não haver.
}

\section{Referências}

1. Dancygier RM, Laitin DD. Immigration into Europe: Economic Discrimination, Violence, and Public Policy. Annu Rev Polit Sci [Internet].2014 May 11 [acesso 2018 Jun 24];17(1):43-64. Disponível em: http://www.annualreviews.org/doi/10.1146/annurev-polisci-082012-115925

2. United Nations. International Migration Report 2017. Econ Soc Aff [Internet]. 2017 [acesso 2018 Jun 29]; Disponível em: http://www. un.org/en/development/desa/population/migration/publications/migrationreport/docs/MigrationReport2017.pdf

3. Brasil. Ministério do Planejamento, Desenvolvimento e Gestão. Instituto Brasileiro de Geografia e Estatística - IBGE. Censo Demográfico 2010 [Internet]. Rio de Janeiro; 2012 [acesso 2018 Jun 28]. Disponível em: https://biblioteca.ibge.gov.br/visualizacao/periodicos/99/ cd_2010_resultados_gerais_amostra.pdf

4. Velasco C, Mantovani F. Em 10 anos, número de imigrantes aumenta 160\% no Brasil, diz PF [Internet]. G1. 2016 [acesso 2018 Jun 24]. Disponível em: http://g1.globo.com/mundo/noticia/2016/06/em-10-anos-numero-de-imigrantes-aumenta-160-no-brasil-diz-pf.html

5. Crimmins EM, Kim JK, Alley DE, Karlamangla A, Seeman T. Hispanic paradox in biological risk profiles. Am J Public Health [Internet]. 2007 Jul [acesso 2018 Jun 24];97(7):1305-10. Disponível em: http://www.ncbi.nlm.nih.gov/pubmed/17538054

6. Gimeno-Feliu LA, Calderón-Larrañaga A, Diaz E, Poblador-Plou B, Macipe-Costa R, Prados-Torres A. The healthy migrant effect in primary care. Gac Sanit [Internet]. 2015 Jan [acesso 2018 Jun 24];29(1):15-20. Disponível em: http://www.ncbi.nlm.nih.gov/pubmed/25176130

7. Kennedy S, Kidd MP, McDonald JT, Biddle N. The Healthy Immigrant Effect: Patterns and Evidence from Four Countries. J Int Migr Integr [Internet]. 2015 May 15 [acesso 2018 Jun 24];16(2):317-32. Disponível em: http://link.springer.com/10.1007/s12134-014-0340-x

8. Edberg M, Cleary S, Vyas A. A trajectory model for understanding and assessing health disparities in immigrant/refugee communities. J Immigr Minor Health [Internet]. 2011 Jun 20 [acesso 2018 Jun 24];13(3):576-84. Disponível em: http://www.ncbi.nlm.nih.gov/ pubmed/20306225

9. United Nations. World Economic Situation and Prospects. New York: United Nations [Internet]; 2016 [acesso 2018 Jun 24]. Disponível em: https://www.un.org/development/desa/dpad/wp-content/uploads/sites/45/publication/2016wesp_full_en.pdf

10. Priebe S, Sandhu S, Dias S, Gaddini A, Greacen T, loannidis E, et al. Good practice in health care for migrants: views and experiences of care professionals in 16 European countries. BMC Public Health [Internet]. 2011 Mar 25 [acesso 2018 Jun 24];11:187. Disponível em: http://www.ncbi.nlm.nih.gov/pubmed/21439059

11. Brasil. Ministério do Planejamento, Desenvolvimento e Gestão. Instituto Brasileiro de Geografia e Estatística - IBGE. Síntese de Indicadores Sociais - Uma análise das condições de vida da população brasileira. Estud e Pesqui Informação Demográfica e Socioeconômica [Internet]. 2016 [acesso 2018 Jun 24];(36). Disponível em: https://biblioteca.ibge.gov.br/visualizacao/livros/liv98965.pdf

12. Gimeno-Feliu LA, Magallón-Botaya R, Macipe-Costa RM, Luzón-Oliver L, Cañada-Millan JL, Lasheras-Barrio M. Differences in the use of primary care services between Spanish national and immigrant patients. J Immigr Minor Health [Internet]. 2013 Jun [acesso 2018 Jun 24];15(3):584-90. Disponível em: http://www.ncbi.nlm.nih.gov/pubmed/22618356 
13. Cavalcanti L, Oliveira AT, Tonhati T, orgs. A inserção dos imigrantes no mercado de trabalho brasileiro. Brasília: Cadernos do Observatório das Migrações Internacionais [Internet]; 2014 [acesso 2018 Jun 24]. p. 1-110. Disponível em: https://oestrangeirodotorg.files.wordpress. com/2014/11/relatorio-parcial-a-inserc3a7ao-dos-imigrantes-no-mercado-de-trabalho-brasileiro.pdf

14. Alessi MLB. A Migração de Haitianos para o Brasil. Conjunt Global [Internet]. 2013 [acesso 2018 Jun 24];2(2):82-6. Disponível em: https:// revistas.ufpr.br/conjgloblal/article/view/35339/21894

15. Sarma S, Simpson W. A microeconometric analysis of Canadian health care utilization. Health Econ [Internet]. 2006 Mar [acesso 2018 Jun 24];15(3):219-39. Disponível em: http://www.ncbi.nlm.nih.gov/pubmed/16229054

16. de Bustillo RM, Antón Jl. Health care utilization and immigration in Spain; 2009 Mar 1 [acesso 2018 Jun 24]. Disponível em: https://mpra. ub.uni-muenchen.de/12382/

17. De Luca G, Ponzo M, Andrés AR. Health care utilization by immigrants in Italy. Int J Health Care Finance Econ [Internet]. 2013 Mar 13 [acesso 2018 Jun 24];13(1):1-31. Disponível em: http://www.ncbi.nlm.nih.gov/pubmed/23239018

18. Carrasco-Garrido P, Jiménez-García R, Barrera VH, de Andrés AL, de Miguel AG. Significant differences in the use of healthcare resources of native-born and foreign born in Spain. BMC Public Health [Internet]. 2009 Jun 25 [acesso 2018 Jun 24];9:201. Disponível em: http:// www.ncbi.nlm.nih.gov/pubmed/19555474

19. Diaz E, Calderón-Larrañaga A, Prado-Torres A, Poblador-Plou B, Gimeno-Feliu LA. How do immigrants use primary health care services? A register-based study in Norway. Eur J Public Health [Internet]. 2015 Feb 1 [acesso 2018 Jun 24];25(1):72-8. Disponível em: http://www. ncbi.nlm.nih.gov/pubmed/25085475

20. Soler-González J, Serna MC, Bosch A, Ruiz MC, Huertas E, Rué M. Sick leave among native and immigrant workers in Spain--a 6-month follow-up study. Scand J Work Environ Health [Internet]. 2008 Dec [acesso 2018 Jun 24];34(6):438-43. Disponível em: http://www.ncbi. nlm.nih.gov/pubmed/19137205

21. Fadnes LT, Møen KA, Diaz E. Primary healthcare usage and morbidity among immigrant children compared with non-immigrant children: a population-based study in Norway. BMJ Open [Internet] 2016 [acesso 2018 Jun 29];6(10):e012101. Disponível em: https://bmjopen. bmj.com/content/6/10/e012101 